\title{
Xiaorong $\mathrm{Li}^{1 \#}$, Jiangning Wang ${ }^{2 \#}$, Haiyun $\mathrm{Shi}^{3}$, Lei Gao ${ }^{4}$, Xueyan Wang*
}

${ }^{1}$ Central Laboratory, The Luhe Teaching Hospital of the Capital Medical University, Beijing, China, ${ }^{2}$ Reparative and reconstructive Sugery, Beijing Shijitan Hospital, Capital Medical University Beijing, China, ${ }^{3}$ Department of Allergy, Beijing Shijitan Hospital, Capital Medical University, Beijing, China, ${ }^{4}$ Plastic Surgery, The Luhe Teaching Hospital of the Capital Medical University, Beijing, China

* Corresponding author, E-mail: xueyanwang135@163.com. " Equal contribution

\begin{abstract}
Background: Radix Scutellariae, a few papers reported its pharmacology activities including alleviate small intestines smooth muscles spasm, sedation, antihypertensive effect. However, the inhibition of its different organic extracts on immediate hypersensitivity has not bee researched.

Materials and Methods: To investigate the anti-immediate hypersensitivity of three extracts including ethanol extracts, acetone extracts, ethyl acetate extracts from Radix Scutellariae, four pharmacological screening model were chose, such as 4-Aminopyridine induced pruritus model, histamine-induced mouse paw edema model, PCA(passive cutaneous anaphylaxis) in ear of mouse, activie cutaneous anaphylaxismouse (mouse ear edema test), furthermore, total IgE level in the sensitized mice serum was evaluated deeply.

Results: Ethanol group at $1.42 \mathrm{~g} / \mathrm{kg}$ and $0.71 \mathrm{~g} / \mathrm{kg}$ could greatly decrease the licking number to 1.2 and 12.7 respectively; also keep mice paw swelling at $0.29 \mathrm{ml}$ and $0.51 \mathrm{ml}$ at $15 \mathrm{~min}$ after injection of histamine. Both ear passive cutaneous allergic reaction and active cutaneous anaphylaxis-ear swelling test demonstrated that ethanol group exhibit great inhibition on immediate hypersensitivity.Low IgE level was found in ethanol group, but high in other two groups.
\end{abstract}

Conclusion: The ethanol extracts exhibits obvious strong inhibition, however, the acetone ones and ethyl acetate showed a little.

Keywords: Radix Scutellariae, Ethanol extracts, Anti-allergic activity, PCA, IgE level

\section{Introduction}

Radix Scutellariae, was well known as the dry root of Scutellaria baicalensis Georgi, a few papers reported its pharmacology activities including alleviate small intestines smooth muscles spasm, sedation, antihypertensive effect. Most importantly, Radix Scutellariae is a excellent anti-allergic TCM in China (Chi et al., 2001). The main action mechanism is was inhibiting the binding of antibody to antigen, destructing mast cell enzyme activation system as well as inhibiting release of histamine from mast cells (M. Bureau et al., 1993).

Some reports showed that the incidence of allergic disease in developed countries was $25 \% \sim 40 \%$, about $30 \%$ in China, this seriously affects the quality of life of patients with allergic disease, and brings great burden to patients, it has now become a public health issue. Fast hypersensitivity was also called type I hypersensitivity and was mediated mainly by main antibody IgE, common diseases including allergic rhinitis, allergic asthma, allergic dermatitis, eczema, urticaria, etc (Zhou et al., 2013).

The main effective component were flavonoids, up to now there are more than 30 compounds, including baicalein, baicalin, chrysin, oroxylin-A, oroxylin-A-7-O-glucuronide, wogonin, wogonoside, skullcaflavone, dihydro baicalin etc(Nagaki et al., 2001), however none reports displayed their activities on anti-allergic application. Thus we finished this research, Kim TW et al has done same work by using a standardized aqueous extract, we conducted three different extracts with different oganic solvents (Kim et al., 2013). Next step we will study the effective compound in antiallergic reaction carefully.

In this study, we revealed that the administration of Radix Scutellariae extract restrained hypersensitivity models. Moreover, to determine the antiallergic inflammatory mechanisms of Radix Scutellariae extracts, we investigated the inhibitory effect of Radix Scutellariae extracts on IgE content in mice sensibilized by diphtheria-tetanus-pertussis (DTP3) vaccine. Our results strongly suggested that Radix Scutellariae ethanol 
http://dx.doi.org/10.4314/ajtcam.v11i5.9

extracts could exhibit satisfactory effects on OVA-induced allergic reaction.

\section{Materials and Methods}

Radix Scutellariae was purchased from Beijing Zi Yun Teng Chinese Herbal Medicine Co., Ltd., evaluated by The College of Traditional Chinese Medicine, Capital Medical University. Kuming mice, male and female are half and half weight from 18 to $22 \mathrm{~g}$, purchased from Capital Medical University, SPF. 75\% ethanol, N-butanol, petroleum ether, 4-aminopyridine (4 -AP), were of analytical grade and Histamine was purchase from Beijing Dong Song Boye Biotechnology Co., Ltd; chlorpheniramine maleate tablets (Beijing Yu Sheng Tang Pharmaceutical Co., Ltd.). Instruments including rotary concentrator (Shanghai Ya Rong Biochemical Instrument Factory), CPA224S electronic analytical balance (Sartorius Corporation), ultrasonic cleaner (Kunshan Shu Mei KQ-250 Uultrasonic Cleaner), micrometer caliper (Grainger), Microplate reader (Thermo).

\section{Preparation of Radix Scutellariae extracts}

$50 \mathrm{~g}$ Radix Scutellariae was crushed and degreased by different volume of petroleum ether $(8 \times, 6 \times, 4 \times)$, extracts were got by backflow extraction with ethanol(70\%), acetone, ethyl acetate $(8 \times, 6 \times, 4 \times)$ according to previous report(Liang et al., 2012).

\section{Drug grouped design}

(1)Blank control, just give double distilled water; (2)ethanol extract group (1.42g/kg, 0.71g/kg, 0.35g/kg); (3) acetone extract group $(1.42 \mathrm{~g} / \mathrm{kg}) ;(4)$ ethyl acetate extract group $(1.42 \mathrm{~g} / \mathrm{kg})$.

\section{4-AP induced allergic skin pruritus test}

60 mice, 18 22g, female and male were half and half, all were grouped into six group. Fasting for four hours and giving the medicine by i.g $2 \mathrm{ml}$ according to the drug grouped design in this paper. One hour later, $1 \mathrm{ml} 4-\mathrm{AP}(0.2 \mathrm{mg} / \mathrm{kg})$ was administered by hypodermic injection at the back of the mouse(Jung et al., 2011). The frequency of licking the application site was recorded every ten minutes, conducting $t$ test. As a result, the anti hypersensitivity activity of the experimental group was compared and screened (Table 1).

Histamine induced paw edema test (Ōyanagui et al., 1981)

70 mice, 18 22g, female and male were half and half, were randomly divided into seven groups. Fasting for four hours and gave the medicine with i.g(intragastric administration) $2 \mathrm{ml}$ according to the drug grouped design amentioned in this paper above, positive control group was given $0.2 \mathrm{ml}, 0.28 \mathrm{mg} / \mathrm{kg}$ chlorpheniramine solution with $i . g$. One hour later, $20 \mu \mathrm{l}$ histamine saline solution $(0.25 \mathrm{mg} / \mathrm{kg})$ were administered at mouse right rear paw by subcutaneous injection, the thickness of mouse right plantar were determined with micrometer calipers before and after the injection of $15 \mathrm{~min}$, 60min respectively. At the same time, swelling degree was expressed by the thickness difference before and after the histamine injection, and all of the data we got were dealed with $t$ test (Table 2).

\section{Mouse ear passive cutaneous allergic reaction (Mican et al., 1992)}

The experimental group were the same as the Histamine induced paw edema test we motioned above. However, we chose the DSCG (disodium cromoglycate) as the positive control group medicine. Female/Male 18 22g KuMing mice were given the Radix Scutellariae Extract suspension in their drinking water for 21 days, according to $0.2 \mathrm{ml}$ solution per grams. The two ear of mice the rats were injected with 1:10 anti-OVA serum $20 \mu \mathrm{L}, 72$ hours later, tail vein $0.25 \mathrm{ml} 0.5 \%$ Evan's Blue solution containing OVA $0.25 \mathrm{mg}$ was injected into tail vein. Half an hour later, kill the mice and got the the auricle (blue parts were cut out). The auricle were put into the test tube, $0.75 \mathrm{ml}, 1 \mathrm{~N} \mathrm{KOH}$ solution was added, $37^{\circ} \mathrm{C}$ overnight digestion. Then add $3.5 \mathrm{ml} 0.6 \mathrm{~N} \mathrm{H}_{3} \mathrm{PO}_{4}$ solution and acetone mixture (mixed according to the proportion of 5:13), extracted 
http://dx.doi.org/10.4314/ajtcam.v11i5.9

by vortex machine shaking, 3000rpm centrifuged for $15 \mathrm{~min}$. The optical density of supernatant was determined at $640 \mathrm{~nm}$ and we then calculate the inhibition rate of PCA (Table 3).

Inhibition Rate $=\frac{\text { blue spot diameter of control group }- \text { blue spot diameter of test group }}{\text { blue spot diameter of control group }} \times 100 \%$

Mice Active Cutaneous Anaphylaxis-Ear swelling test (Teshima et al., 1998)

The administration of the extracts and control group were the same as the above test. However, the positive control medicine DSCG should be gave, i.g $0.1 \mathrm{~g} / \mathrm{kg} /$ day. $200 \mu \mathrm{g}$ OVA and $0.2 \mathrm{ml}$ Diphtheria pertussis tetanus vaccine were given the animal by i.g in the day before the test, and $8 \mathrm{mgAI}(\mathrm{OH})_{3}$ were given in the fouth day, then waiting for three weeks to make the animal sensitized. $20 \mu \mathrm{L}$ (containing $50 \mu \mathrm{g}$ OVA) were injected into the left ear subcutaneously, the same volume of physiological saline into the right ear. Binaural thickness were determined $20 \mathrm{~min}$ later, calculated the thickness difference and the percentage of swelling inhibition (Table 4).

Determination of total IgE in serum of mice (Jung et al., 2012)

Compared with the above test, this part have another group, normal control group, the other process was the same as the above part, however, the sensitization time was 2 weeks, and picked the blood from eyeball separation blood serum. The whole operation conducted according to the method of operation in the kit (Table 5 and Figure 2).

\section{Results}

\section{Anti-4-AP induced allergic skin pruritus test}

Took a animal model of allergic skin pruritus for efficacy trials of RADIX SCUTELLARIAE extract (Table 1).

Table 1: Anti-itching effect of RADIX SCUTELLARIAE extract

\begin{tabular}{ccl}
\hline Group & Dose $(\mathbf{g} / \mathbf{k g})$ & $\mathbf{L F}(\mathbf{X} \pm \mathbf{s})$ \\
\hline Blank control & - & $38.60 \pm 5.35$ \\
Ethanol group & 0.35 & $18.24 \pm 5.89$ \\
& 0.71 & $12.70 \pm 4.21^{* *}$ \\
Acetone group & 1.42 & $1.20 \pm 1.10^{* *}$ \\
Ethyl acetate group & 1.42 & $18.14 \pm 5.10$ \\
\hline
\end{tabular}

Notes: ** indicated that $\mathrm{P}<0.01$ (compared with blank control group); LF means licking frequency.

We can read from the Table 1 that the licking number has no obvious difference between acetone group and ethyl acetate group, indicating that the extracts resulted from this two kind of solvent could not exhibit big efficiency. In the ethanol group, the administration of the medicine at the concentration of $1.42 \mathrm{~g} / \mathrm{kg}$ and $0.71 \mathrm{~g} / \mathrm{kg}$ could greatly decrease the licking number, and has a significant difference when compared with blank group, indicating that $0.71 \mathrm{~g} / \mathrm{kg}$ of ethanol extracts could be the effective threshold dose on anti allergic itching effect. Further more, the effect would be stronger as the dose of i.g become more and more bigger. 
http://dx.doi.org/10.4314/ajtcam.v11i5.9

Anti-histamin induced paw swelling effect

Table 2: Effect of extracts on mice paw swelling $(\bar{X} \pm s, n=10)$

\begin{tabular}{cccc}
\hline Group & $\begin{array}{c}\text { Dose } \\
(\mathbf{g} / \mathbf{k g})\end{array}$ & $\mathbf{1 5 m i n}$ & $\mathbf{6 S}\left(\mathbf{1 0}^{-\mathbf{2}} \mathbf{m l}\right)$ \\
& - & $78.9 \pm 7.24$ & $45.4 \pm 5.16$ \\
Blank control & 0.35 & $65.5 \pm 8.7$ & $40.2 \pm 6.8$ \\
Ethanol & 0.71 & $51.2 \pm 4.4^{*}$ & $27.2 \pm 7.3^{* *}$ \\
& 1.42 & $29.1 \pm 3.2^{* *}$ & $25.6 \pm 5.3^{* *}$ \\
Acetone & 1.42 & $43.2 \pm 8.5^{* *}$ & $38.9 \pm 5.9^{*}$ \\
Ethyl acetate & 1.42 & $42.4 \pm 7.8^{* *}$ & $34.5 \pm 5.2^{*}$ \\
\hline
\end{tabular}

Notes: * indicated $P<0.05$, ** indicated $P<0.01$, **( compared with blank control); PS means paw swelling.

The effect of extracts on mice paw swelling could be seen in Table 2 . The concentration of $1.42 \mathrm{mg} / \mathrm{kg}$ in ethanol group could greatly inhibit the paw swelling, the higher concentration, the more obvious effect. Compared with the ethanol group, the acetone group and the ethyl acetate group could get to the same or higher efficiency at the same concentration.

\section{Mouse ear passive cutaneous allergic reaction}

Table 3: Mouse ear passive cutaneous allergic reaction

\begin{tabular}{cccc}
\hline Group & Dose $(\mathbf{g} / \mathbf{k g})$ & OD value & IR \\
& & $\mathbf{A 6 4 0} \mathbf{n m}(\overline{\mathbf{X}} \pm \mathbf{S})$ & $\mathbf{( \% )}$ \\
\hline Negative control & - & $0.058 \pm 0.016$ & - \\
Ethanol extracts & 0.35 & $0.046 \pm 0.023$ & 20.69 \\
& 0.71 & $0.039 \pm 0.012^{*}$ & 32.76 \\
Acetone extracts & 1.42 & $0.026 \pm 0.024^{* *}$ & 55.17 \\
Ethyl acetate extracts & 1.42 & $0.041 \pm 0.015^{* *}$ & 29.31 \\
\hline
\end{tabular}

Notes: * indicated that $p<0.05, * *$ indicated that $p<0.01$, when compared with negative control; Dose $(g / k g)$ means Crude drugs/ mice weight; IR meaans inhibition rate.

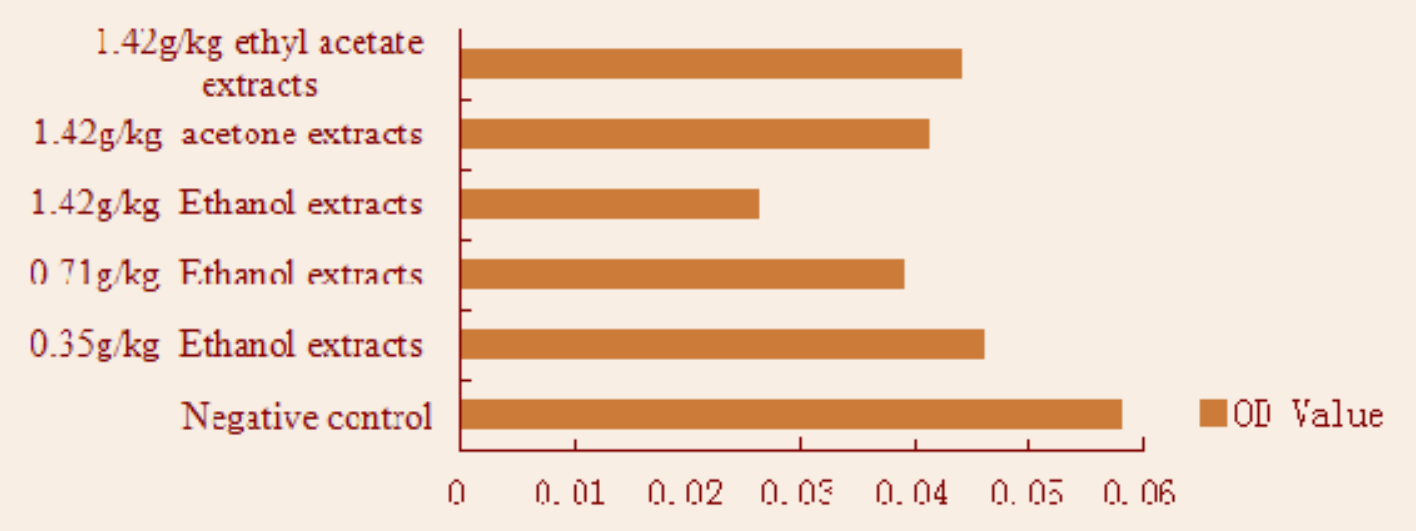

Figure 1 Mouse ear passive cutaneous allergic reaction 
http://dx.doi.org/10.4314/ajtcam.v11i5.9

We can read from Figure 1 and Table 4 that the highest anti-allergic effect in PCA belonged to high concentration of ethanol extracts, and the inhibition rate was $55.17 \%$.

Mice Active Cutaneous Anaphylaxis-Ear swelling test

Table 4: Results of Ear Swelling Test

\begin{tabular}{ccccc}
\hline Group & $\begin{array}{c}\text { Dose } \\
(\mathbf{g} / \mathbf{k g})\end{array}$ & AN & $\mathbf{E S}(\mathbf{m m})$ & SIR(\%) \\
\hline Negative control & - & 10 & $2.59 \pm 0.92$ & - \\
Cromolyn sodium & 0.1 & 10 & $1.75 \pm 0.62^{*}$ & 32.43 \\
Ethanol extracts & 0.35 & 10 & $1.56 \pm 0.91^{*}$ & 39.77 \\
& 0.71 & 10 & $1.45 \pm 0.55^{*}$ & 44.02 \\
Acetone extracts & 1.42 & 10 & $1.37 \pm 0.63^{* *}$ & 47.10 \\
ethyl acetate extracts & 1.42 & 10 & $1.62 \pm 0.37^{*}$ & 37.45 \\
\hline
\end{tabular}

Notes: * indicated that $p<0.05, * *$ indicated that $p<0.01$, when compared with negative control; AN means animal number; ES means ear swelling; SIR means swelling inhibition rate.

Determination of total IgE in serum of mice

Table 5: Total IgE in serum of mice

\begin{tabular}{lcc}
\hline Group & Dose $(\mathbf{g} / \mathbf{k g})$ & IgE level $(\mathbf{~ I U} / \mathbf{m L})$ \\
\hline Normal control & 0.1 & $8.57 \pm 1.18^{*}$ \\
Negative control & - & $25.21 \pm 2.36$ \\
Ethanol extracts & 0.35 & $8.12 \pm 1.54 *$ \\
& 0.71 & $5.73 \pm 1.02 *$ \\
Acetone extracts & 1.42 & $3.23 \pm 1.05^{* *}$ \\
ethyl acetate extracts & 1.42 & $8.42 \pm 1.56$ \\
Sodium cromoglycate & 1.42 & $8.51 \pm 1.03$ \\
\hline
\end{tabular}

Notes: * indicated that $p<0.05, * *$ indicated that $p<0.01$, when compared with negative control.

We can read from the Figure 2 and Table 4 that the ethanol extracts could decrease the IgE content in serum of mice at a very low level. Furthermore, the concentration of drug has a positive relationship with decreased IgE, however, the IgE level in acetone group or in ethyl acetate group could not reach the same effect, but the effect of $1.42 \mathrm{~g} / \mathrm{kg}$ in acetone/ethyl acetate group equals to the effect of $0.35 \mathrm{~g} / \mathrm{kg}$ in ethanol group. 


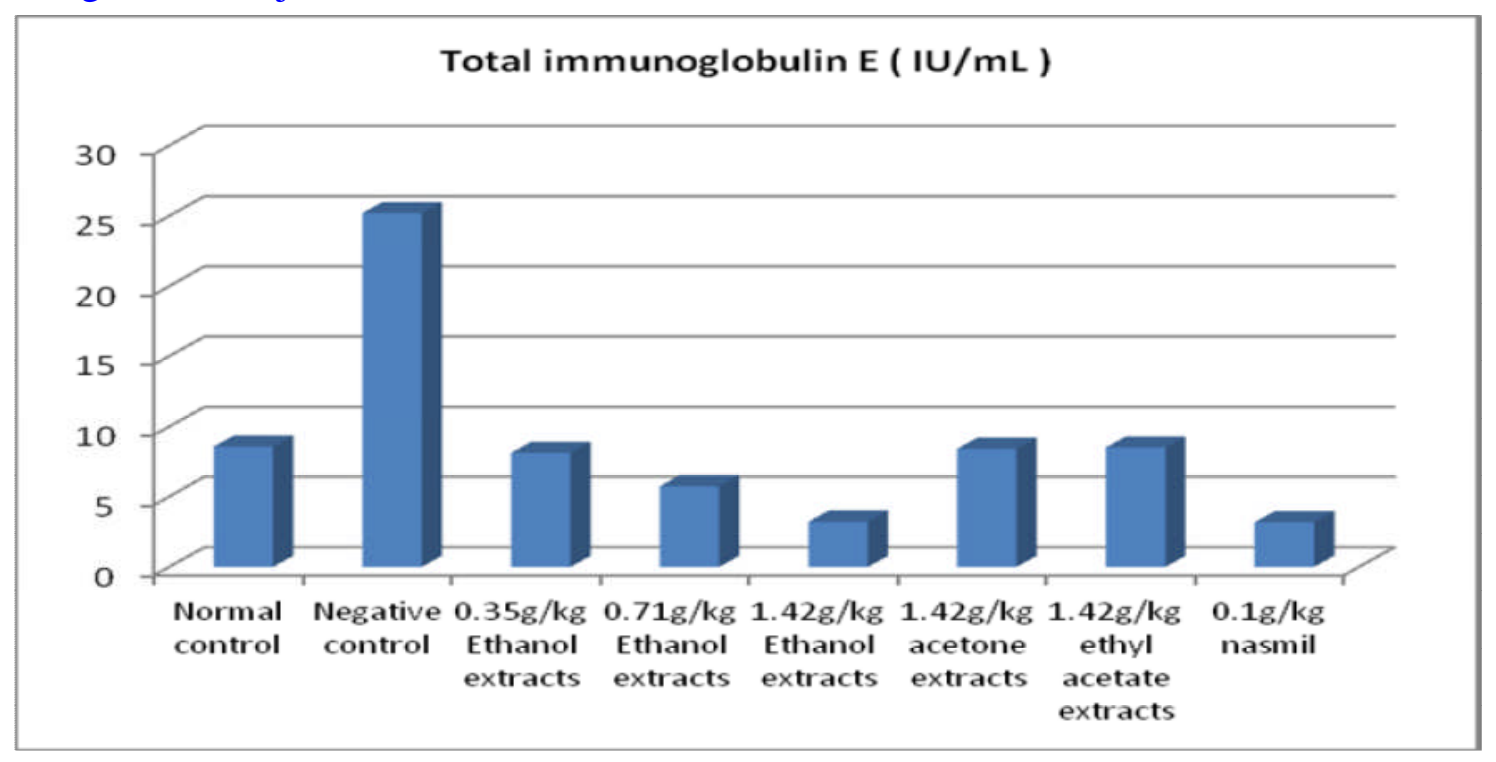

Figure 2: Total IgE level in serum of mice

\section{Discussion}

This research has conducted a broad pharmacology activity screen on the extracts got from the TCM Radix Scutellariae, including ethanol extracts with different concentration, acetone extracts and ethyl acetate extracts. The pharmacology tests including anti paw edema induced by histamine, anti-PCA in mouse ear and anti-ACA in mouse ear, we also determine the total IgE level in serum of mice.

Using the ELISA-sandwich technique in this research to determine the IgE level in serum of sensitized mice, results showed that the ethanol extracts group have a significant difference with the control model group, indicated that extracts of Radix Scutellariae could decrease the IgE level in serum of sensitized mice and decrease the sensitivity of type I hypersensitivity, so this extracts could prevent type I hypersensitivity at a low dose of administration, but as for the mechanism of action, we need to take a deep research on it in future. Otherwise, in the treatment of acute eczema- one of the disease belonged to type I hypersensitivity, the 'Sanwu Huangqin Granules' being as a important effective and valuable TCM in China, most of all, Radix Scutellariae was a main ingredient, thus we chose it as our research objective, inquiring into the action of it on immediate hypersensitivity, in order to supply more useful data and strong basis for the development of anti-allergy drugs (Zhang et al., 2008).

We observed the effect of extracts on immediate-type hypersensitivity, found that the ethanol extracts could exhibited the best efficiency in the above three pharmacology model test, indicating that the inhibition mechanism of the extracts may be have a revelance with histamine decrease and other mediators of inflammation decrease. All of the results we got in this research could provide a basis for the treatment of the skin pruritus, urticaria, eczema or other diseases induced by immediate-type hypersensitivity (Hyungwoo et al., 2013).

Results showed that the ethanol extracts of Radix Scutellariae had a obvious inhibition effect on the pruritus in mice, meant that the extracts had antipruritic effect, and the 4-AP induced pruritus model was related to histamine level. It is known that H1 receptor was found in sensory nerve ending which was combined with histamine would produce itching feeling in humanbody. 4-AP is a potassium channel blocker, could block potassium channels, decrease membrane potential, then calcium channel was easy to open, more ions influxed, promoting the release of neurotransmitters from nerve endings, increased mast cell degranulation and released histamine, at last induced skin pruritus (Feinberg et al., 1946).

We could infer that antipruritic effect of ethanol extracts of Radix Scutellariae may be related to its antihistamine effect. At the same time, we found that the extracts had a obvious effect on the PCA and ACA model which was famous for screening novel anti-allergic substances, saying that the extracts of Radix Scutellariae had possibility on the new antiallergic drug research and development.

In the next step, we will compared the ethanol extracts and the aqueous extracts reported by Kim Ms et al. Then, make a deep purification and isolation on it, in order to find a highly active compound from Radix Scutellariae by using HPLC and column chromatography. 


\section{Acknowledgment}

We thank the allergy labaratory in Beijing Shijitan Hospital,CMU for their help in analysing the results.

Conflict of interests: We have no conflict of interest.

\section{References}

1. Baoguo Zhang, Xiaoxia Liang, Qingfang Liu. (2008). Modern pharmacodynamics research and clinic application of Huangqintang. China Journal of Chinese Materia Medica. 33(22):2587-2590.

2. Hyuk-Sang Jung, Mi Hye Kim, Nam-Gil Gwak, Yong-Seok Im, Ka-Yeon Lee, Youngjoo Sohn, Hyeon Choi, Woong Mo Yang. (2012). Antiallergic effects of Scutellaria baicalensis on inflammation in vivo and in vitro. J Ethnopharmacol. 141(1):345-349.

3. Hyungwoo Kim, Miyoung Kim, Hanna Kim, Guem San Lee, Won Gun An, Su In Cho. (2013). Anti-inflammatory activities of Dictamnus dasycarpus Turcz., root bark on allergic contact dermatitis induced by dinitrofluorobenzene in mice. J Ethnopharmacol. 149(2):471-477.

4. Jo Ann M. Mican, Naveen Arora, Parris R. Burd, Dean D. Metcalfe. (1992). Passive cutaneous anaphylaxis in mouse skin is associated with local accumulation of interleukin-6 mRNA and immunoreactive interleukin-6 protein. J Allergy Clin Immun. 90(5):815-824.

5. Jung, H.W., Jung, J.K., Park, Y.K. (2011). Anti-allergic effect of Ostericum koreanum root extract on ovalbumin-induced allergic rhinitis mouse model and mast cells. Asian Pac J Allergy Immunol 29(4):338-48.

6. Kim, T.W., Song, I.B., Lee, H.K., Kim, M.S., Ham, S.H., Cho, J.H., Lim, J.H., Yun, H.I. (2013). Assessment of dermal safety of Scutellaria baicalensis aqueous extract topical application on skin hypersensitivity. Planta Med.79 (11):959-62.

7. Liang, X.L., Liao, Z.G., Zhu, J.Y., Zhao, G.W., Yang, M., Yin, R.L., Cao, Y.C., Zhang, J., Zhao, L.J. (2012). The absorption characterization effects and mechanism of Radix Angelicae dahuricae extracts on baicalin in Radix Scutellariae using in vivo and in vitro absorption models. J Ethnopharmacol. 139(1):52-7.

8. M. Bureau, E. Coëfier, S. Desquand, J. Lefort, M.A. Martins, M. Pretolani, P. Silva, D. Vincent, B.B. Vargafti. (1993). Mechanisms of Immediate Hypersensitivity and Hyper-responsiveness: Mediators, Antagonists and Role of Interleukins. T-Lymphocyte and Inflammatory Cell Research in Asthma, Academic Press, San Diego. 155-166.

9. Reiko Teshima, Hiroshi Akiyama, Reiko Akasaka, Yukihiro Goda, Masatake Toyoda, Jun-ichi Sawada. (1998). Simple spectrophotometric analysis of passive and active ear cutaneous anaphylaxis in the mouse. Toxicol lett. 95(2):109-115.

10. Samuel M. Feinberg. (1946). Histamine antagonists;Summary of developments in nonspecific inhibition of histamine, anaphylaxis, and allergy. J Allergy. 17(4):217-230.

11. Yasunori Nagaki, Seiji Hayasaka, Chiharu Kadoi, Nobuo Nakamura, Yoriko Hayasaka. (2001). Effects of Scutellariae Radix Extract and its Components (Baicalein, Baicalin, and Wogonin) on the Experimental Elevation of Aqueous Flare in Pigmented Rabbits. JPN J Ophthalmol. 45(3):216-220.

12. Yeon Sook Chi, Bong Sun Cheon, Hyun Pyo Kim. (2001). Effect of wogonin, A plant flavone from Scutellaria radix, on the suppression of cyclooxygenase-2 and the induction of inducible nitric oxide synthase in lipopolysaccharide-treated RAW 264.7 cells. Biochem Pharmacol. 61(10): $1195-1203$.

13. Yoshihiko Ōyanagui. (1981). Steroid-like anti-inflammatory effect of superoxide dismutase in serotonin-, histamine- and kinin-induced edemata of mice: Existence of vascular permeability regulating protein(S). Biochem Pharmacol. 30(13):1791-1798.

14. Zhou, J.S., Sandomenico, A., Severino, V., Burton, O.T. (2013). Darling A, Oettgen HC, Ruvo M. An IgE receptor mimetic peptide (PepE) protects mice from IgE mediated anaphylaxis. Mol Biosyst. 9(11):2853-2859. 